\title{
Just noticeable difference in olfaction is related to trigeminal component of odorants*
}

\author{
L. Jacquot, J. Hidalgo, G. Brand \\ Laboratoire de Neurosciences, Université de Franche-Comté, Place Leclerc 25000 Besançon, France
}

\begin{abstract}
SUMMARY Many studies have dealt with the interrelationships between both olfactory and trigeminal systems but a poorly explored question concerns the role of each system in the detection processes, especially in the just noticeable difference (JND). The aim of this study was to investigate variations in JNDs for three odorants in relation to their trigeminal component, i.e. low, middle, high. The results indicated that the higher the trigeminal component, the lower the JND, suggesting a better capacity to perceive intensity changes for pungent odorants than for relatively pure odorants.
\end{abstract}

Key words: trigeminal nerve, olfactory perception, just noticeable difference, sex differences

\section{INTRODUCTION}

In the nasal cavity, the capacity to detect and react to volatile chemicals is mediated by two anatomically separated sensory pathways, the olfactory and the trigeminal systems. However, both systems are known to interact ${ }^{(1)}$ at different levels of the chemoreception processes. Olfactory receptors (CN I) positioned in the upper recesses of the nasal cavity coexist with free nerve endings of the ophthalmic and maxillary branches of the trigeminal nerve $(\mathrm{CN} \mathrm{V})$ distributed throughout the nasal mucosa and the olfactory epithelium ${ }^{(2)}$. Most volatile molecules, at sufficient concentration, elicit both olfactory and trigeminal activations ${ }^{(3,4)}$ although the pungent properties appear to be strongly different from a molecule to another ${ }^{(5)}$.

For a long time ${ }^{(6,7)}$, many studies have investigated the interrelationships between olfactory and trigeminal systems and have contributed to a better understanding of transduction, perception and cortical treatment of nasal stimuli. It was generally suggested that the trigeminal nerve influenced olfactory afferent inputs but in some not clearly defined manner. Moreover, the role of both systems in detection processes has been poorly explored ${ }^{(8-10)}$. For instance, it has been demonstrated that unconscious odour detection could be due to the trigeminal component of odorant ${ }^{(11)}$ and that a pre-stimulation of the trigeminal nerve modified the subsequent olfactory sensitivity ${ }^{(12)}$. However, the published works had used preferentially the absolute detection thresholds over the differential threshold.

In sensory perception, the differential threshold also called just noticeable difference (JND), is defined by the level at which an increase in a detected stimulus can be perceived or the smallest change in stimulation that a subject can detect. JND might be a more sensitive measure for assessing the quality of olfactory perception in different manners such as comparative assessment between a single molecule and mixtures ${ }^{(13)}$, clinical test ${ }^{(14)}$ or damages to the olfactory system ${ }^{(15)}$, than the most frequently measured absolute threshold ${ }^{(16)}$.

However, the role of the trigeminal component of an odorant in the JND has never been investigated. Thus, the aim of the present study was to compare in the same population the JNDs obtained with nasal stimuli chosen in relation to their trigeminal activation properties, i.e. low, middle and high and previously used in classical absolute detection thresholds ${ }^{(11)}$. Additionally, as olfactory perception is well known to depend on gender ${ }^{(17)}$, male and female subjects were compared in the present study.

\section{METHODS}

Subjects

Forty volunteer subjects participated in this experiment, 20 males and 20 females. Their age ranged from 22 to 29 years (mean age 25 years 7 months). All subjects were non-smokers and reported normal smell sensitivity. None of them had a history of nasal/sinus disease or extensive exposure to chemicals with potential toxicity. The study was conducted in accordance with the Declaration of Helsinki - Hong Kong.

\section{Odorants}

Three specific odorants (Table 1) were used in relation to their trigeminal properties ${ }^{(5)}$ : Phenyl ethyl alcohol (PEA) with very low intranasal trigeminal properties, butanol (BUT) with middle trigeminal properties and pyridine (PYR) with high trigeminal properties. Dilutions series (factor 2) were prepared in 
Table 1. Properties of phenyl ethyl alcohol (PEA), butanol (BUT) and pyridine (PYR).

\begin{tabular}{lcccccc}
\hline Chemical & Company & CAS* & $\begin{array}{c}\text { Molecular } \\
\text { formula }\end{array}$ & Mol. wt & $\begin{array}{c}\text { Density } \\
\mathrm{g} / \mathrm{cm}^{3}\end{array}$ & $\mathrm{~mol} / \mathrm{cm}^{3}$ \\
\hline Phenyl ethyl alcohol & Sigma & $60-12-8$ & C8H10O & 122.2 & 1.02 & $8.34 \times 10^{-3}$ \\
Butanol & Sigma & $71-36-3$ & C4H10O & 74.12 & 0.81 & $10.9 \times 10^{-3}$ \\
Pyridine & Sigma & $110-86-1$ & C5H5N & 79.10 & 0.98 & $12.4 \times 10^{-3}$ \\
\hline
\end{tabular}

*The American Chemical Society's Chemical Abstracts Service (CAS) registry number

deionized water for each chemical product. After successive dilutions (Table 2), the full series included steps 1 to 11 (step 1 as the highest concentration). Four $\mathrm{mL}$ of each concentration were placed in a glass tube $(7.5 \mathrm{~cm}$ high, $1 \mathrm{~cm}$ in diameter at the opening. Step 6 was used as the reference concentration.

\section{Procedure}

Ten concentrations (steps 1 to 5 and steps 7 to 11) were presented in a randomized order into 5 series repeated twice. A full experiment with an odorant consisted in a total of 100 trials with 10 trials for each stimulus comparison. A rest period of 2 minutes was observed between the series. The full experiment for an odorant lasted about 1 hour. Each odorant was tested in a specific session and the sessions (in a randomized order) were separated with a delay of one week. All concentration steps were compared to step 6 (i.e. reference concentration) in a classical two alternative forced-choice task. The subject had to indicate whether the concentration in the test tube was higher or lower than the reference one.

\section{Analyses}

The differential threshold was calculated by using the following two equations commonly performed to obtain upper and lower limits ${ }^{(18)}$. For each concentration tested, the discrimination threshold was defined as the stimulus magnitude of the comparison at which the proportion of correct responses was equal to 0.75 .

$$
\begin{array}{ll}
\text { Upper } & S_{U}=\left(C_{U}+\frac{D}{2}\right)-\frac{\sum_{i=1}^{p} U_{i}}{n} D \\
\text { Lower } & S_{L}=\left(C_{L}+\frac{D}{2}\right)+\frac{\sum_{i=1}^{p} L_{i}}{n} D \\
\text { Differential Threshold: } & \Delta L=\frac{S_{V}-S_{L}}{2}
\end{array}
$$

$C_{U}$ : Maximum stimulus compared, $C_{L}$ : Minimum stimulus compared, $U i$ : Total number of judgments that belong to the high limit in the " ${ }^{\text {th }}$ stimulus comparison, $L_{i}$ : Total number of judgments that belong to the "low" limit in the " $i$ "th stimulus comparison, $n$ : Number of trials performed in each stimulus comparison, $D$ : Step size of comparison stimulus, $p$ : Total number of comparison stimulus.

\section{RESULTS}

Results are reported in Figure 1. A 2 (sex) x 3 (odour) ANOVA with repeated measures showed no significant gender effect $[\mathrm{F}(1.39)=2.68, \mathrm{~ns}]$, but significant differences in relation to odorant tested $[\mathrm{F}(2.78)=56.7, \mathrm{p}<0.0001]$ and a significant interaction between both factors $[\mathrm{F}(2.78)=4.65$, $\mathrm{p}<0.01]$. Post hoc Scheffé tests showed that JND was higher for PEA ( $\mathrm{m}=$ $2.81, \mathrm{sd}=0.14)$ than for BUT $(\mathrm{m}=2.12, \mathrm{sd}=0.10)[\mathrm{F}=12.56$, $\mathrm{p}<0.05]$, higher for PEA than PYR $(\mathrm{m}=1.77$, $\mathrm{sd}=0.07)$ $[\mathrm{F}=28.88, \mathrm{p}<0.05]$ and higher for BUT than for PYR $[\mathrm{F}=3.35, \mathrm{p}<0.05]$. Moreover, a significant difference related to sex was only observed for PEA $[\mathrm{F}=4.19, \mathrm{p}<0.04]$ with a higher JND for the male group $(\mathrm{m}=3, \mathrm{sd}=0.21)$ than for the female group $(\mathrm{m}=2.62, \mathrm{sd}=0.16)$.

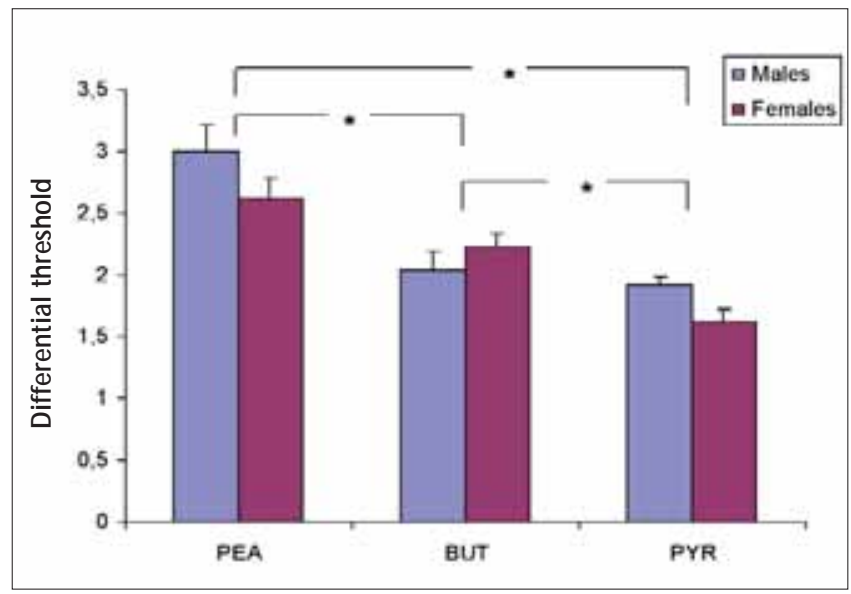

Figure 1. Differential thresholds obtained in male $(\mathrm{N}=20)$ and female $(\mathrm{N}=20)$ groups with three odorants, phenyl ethyl alcohol (PEA), butanol (BUT) and pyridine (PYR). $\left({ }^{*}\right) \mathrm{p}<0.05$.

\section{DISCUSSION}

The results of the present study indicate that the stronger the trigeminal component, the lower the JND, suggesting a better capacity to perceive intensity changes for pungent odorants than for relatively pure odorants. These findings are in agreement with data obtained for absolute thresholds with the same molecules ${ }^{(11)}$ concerning significant differences between the relatively pure odorant PEA and mixed olfactory/trigeminal odorants BUT and PYR. In both cases, nasal chemical sensitivity measured psychophysically, appeared better with the mixed odorants. However, in the present study the JND differences observed between BUT and PYR were not revealed in the case of absolute thresholds. These findings reinforce the hypothesis 
Table 2. Concentrations of phenyl ethyl alcohol, butanol and pyridine obtained by successive dilutions (factor two).

\begin{tabular}{|c|c|c|c|c|c|c|c|}
\hline \multirow[b]{2}{*}{$\begin{array}{l}\text { Dilution } \\
\text { step }\end{array}$} & \multirow[b]{2}{*}{$\begin{array}{c}\text { Concentration } \\
(\% \mathrm{v} / \mathrm{v})\end{array}$} & \multicolumn{2}{|c|}{ phenyl ethyl alcohol } & \multicolumn{2}{|c|}{ butanol } & \multicolumn{2}{|c|}{ pyridine } \\
\hline & & $\mathrm{g} / \mathrm{cm}^{3}$ & $\mathrm{~mol} / \mathrm{cm}^{3}$ & $\mathrm{~g} / \mathrm{cm}^{3}$ & $\mathrm{~mol} / \mathrm{cm}^{3}$ & $\mathrm{~g} / \mathrm{cm}^{3}$ & $\mathrm{~mol} / \mathrm{cm}^{3}$ \\
\hline 1 & 100 & 1.02 & $8.34 \times 10^{-3}$ & 0.81 & $10.9 \times 10^{-3}$ & 0.98 & $12.4 \times 10^{-3}$ \\
\hline \multicolumn{8}{|c|}{ Pure liquid } \\
\hline 2 & 50 & 0.51 & $4.17 \times 10^{-3}$ & 0.405 & $5.45 \times 10^{-3}$ & 0.49 & $6.2 \times 10^{-3}$ \\
\hline 3 & 25 & 0.255 & $2.085 \times 10^{-3}$ & 0.2025 & $2.725 \times 10^{-3}$ & 0.245 & $3.1 \times 10^{-3}$ \\
\hline 10 & 0.195 & $1.989 \times 10^{-3}$ & $1.626 \times 10^{-5}$ & $1.579 \times 10^{-3}$ & $2.125 \times 10^{-5}$ & $1.911 \times 10^{-3}$ & $2.418 \times 10^{-5}$ \\
\hline
\end{tabular}

suggesting that the JND could be a more discriminative tool than the absolute threshold and point out the role of the trigeminal system in a greater ability to detect and discriminate intensity variations of volatile chemicals. This fact could be related to a functional point of view. Indeed, the olfactory system is mainly involved in the identification and recognition of a large variety of molecules while the non-selective trigeminal system is clearly involved in physiological mechanisms devoted to protect respiratory and gustatory tracts, because many toxic substances are associated with pungency. Thus, from an adaptative point of view, it appears more efficient to have a greater ability to detect molecules in relation to their noxiousness.

It must be noted that the hedonic valence varied according to the molecules used, i.e. pleasant for PEA, neutral for BUT and unpleasant for PYR. However, it has been previously demonstrated ${ }^{(19)}$ that the hedonic quality was not predominant in psychophysical and psychophysiological responses to odorants. Therefore, the JNDs observed in the present work can be mainly related to the trigeminal component of odorants.

The findings of the present study also showed differences related to sex in the JND for PEA but not for BUT and PYR suggesting that the greater ability in detection processes for women is dependent of the olfactory system while the trigeminal system is not gender discriminative. Sex differences in olfaction ${ }^{(17)}$ are well known and extensively described but sex differences related to the trigeminal component of odorants appeared as an unsolved question. Some studies have failed to find significant sex differences in nasal irritation ratings ${ }^{(3,20)}$, whatever the age ${ }^{(21)}$ while other studies reported sex differences in detection thresholds ${ }^{(22)}$ and electrophysiological responses ${ }^{(23-25)}$. Additionally, trigeminal detection is lateralized while no such phenomenon exists in olfactory detection ${ }^{(26)}$. Studies using lateralized tasks found no sex differences in trigeminal sensitivity ${ }^{(27,28)}$. It would be relevant in further research to compare such lateralized tasks in unilateral JND thresholds according to the trigeminal component of odorants in addition to previous works focused on olfactory/trigeminal lateralization processes ${ }^{(29)}$. In the same way, as JND appears as a more sensitive measure than the absolute threshold, further research could also consider this measure in clinical popula- tions insofar as the intranasal trigeminal function is different in the presence or absence of an intact sense of smell ${ }^{(30)}$, especially in neurodegenerative disease, i.e. Alzheimer or Parkinson disease, in which olfactory sensitivity is known to be early strongly disrupted ${ }^{(31,32)}$.

\section{REFERENCES}

1. Brand G. Olfactory/trigeminal interactions in nasal chemoreception. Neurosci Biobehav Rev. 2006; 30: 908-917.

2. Lang J. Clinical Anatomy of the Nose, Nasal Cavity and Paranasal Sinuses. Thieme, Stuttgart, 1989.

3. Cain WS, Murphy CL. Interaction between chemoreceptive modalities of odour and irritation. Nature. 1980; 284: 255-257.

4. Cometto-Muniz JE, Hernandez S. Odorous and pungent attributes of mixed and unmixed odorants. Percept Psychophys. 1990; 47: 391-399.

5. Doty RL, Brugger WPE, Jurs PC, et al. Intranasal trigeminal stimulation from odorous volatiles: psychometric responses from anosmics and normal humans. Physiol Behav. 1978; 20: 175-185.

6. Stone H, Rebert CS. Observations on trigeminal olfactory interactions. Brain Res. 1970; 21: 138-142.

7. Stone H, Williams B, Carregal EJA. The role of the trigeminal nerve in olfaction. Exp Neurol. 1968; 21: 11-19.

8. Cometto-Muniz JE, Cain WS. Thresholds for odor and nasal pungency. Physiol Behav. 1990; 48: 719-725.

9. Cometto-Muniz JE, Cain WS. Trigeminal and olfactory sensitivity: comparison of modalities and methods of measurement. Int Arch Occup Environ Health. 1998; 71: 105-110.

10. Hummel T. Assessment of intranasal trigeminal function. Int $\mathrm{J}$ Psychophysiol. 2000; 36: 147-155.

11. Jacquot L, Monnin J, Brand G. Uncouscious odor detection could not be due to odor itself. Brain Res. 2004; 1002: 51-54.

12. Jacquot L, Monnin J, Brand G. Influence of nasal trigeminal stimuli on olfactory sensitivity. CR Biologies, 2004; 327: 305-311.

13. Le Berre E, Béno N, Ishii A, Chabanet C, Etiévant P, ThomasDanguin T. Just noticeable differences in component concentrations modify the odor quality of a blending mixture. Chem Sens. 33, 389-395.

14. Eichenbaum H, Morton TH, Potter H, et al. Selective olfactory deficits in case H.M. Brain. 1983; 106: 459-472.

15. Slotnik BM, Bell GA, Panhuber H, et al. Detection and discrimination of proprionic acid after removal of its 2-DG identified major focus in the olfactory bulb: a psychophysical analysis. Brain Res. 1997; 762: 89-96.

16. Doty RL. Handbook of olfaction and gustation. Marcel Dekker, New York, 2003.

17. Brand G, Millot JL. Sex differences in human olfaction: between evidence and enigma. Quart J Exp Psychol. 2001; 54B: 259-270.

18. McKee S, Klein SA, Teller DY. Statistical properties of forcedchoice psychometric functions: Implications of probit analysis. Percep Psychophys. 1985; 37: 286-298.

19. Brand G, Jacquot L. Quality of odor and olfactory lateralization processes in humans. Neurosci Lett. 2001; 316: 91-94. 
20. Medina MRG, Cain WS. Bilateral integration in the common chemical sense. Physiol Behav. 1982; 29: 349-353.

21. Stevens JC, Plantinga A, Cain WS. Reduction of odor and nasal pungency associated with aging. Neurobiol Aging. 1982; 3: 125132.

22. Dunn JD, Cometto-Muniz JE, Cain WS Nasal reflexes: Reduced sensitivity to $\mathrm{CO} 2$ irritation in cigarette smokers. J Appl Toxicol. 1982; 2: 176-178.

23. Hummel T, Barz S, Pauli E, et al. Chemosensory event-related potentials change as a function of age. Electroencephalogr Clin Neurophysiol. 1998; 108: 208-217.

24. Olofsson JK, Nordin S. Gender differences in chemosensory perception and event-related potentials. Chem Sens. 2004; 29: 629637.

25. Lundström JN, Frasnelli J, Larsson M, et al. Sex differentiated responses to intranasal trigeminal stimuli. Int $\mathrm{J}$ Psychophysiol. 2005; 57: 181-186.

26. Frasnelli J, Charbonneau G, Collignon O, et al. Odor localization and sniffing. Chem Sens. 2009; 34: 139-144.

27. Hummel T, Futschik T, Frasnelli J, et al. Effects of olfactory function, age and gender on trigeminally mediated sensations: a study based on the lateralization of chemosensory stimuli. Toxicol Lett. 2003; 140: 273-280.

28. Wysocki CJ, Cowart BJ. Nasal trigeminal chemosensitivity across the life span. Percept Pychophys. 2003; 65: 115-122.
29. Brand G, Millot JL, Saffaux M, et al. Lateralization in human nasal chemoreception: differences in bilateral electrodermal responses related to olfactory and trigeminal stimuli. Behav Brain Res. 2002; 133: 205-210.

30. Iannilli E, Gerber J, Frasnelli J, et al. Intranasl trigeminal function in subjects with and without an intact sense of smell. Brain Res. 2007; 1139: 235-244.

31. Barrios FA, Gonzales L, Favila R, et al. Olfaction and neurodegeneration in HD. Clin Neurosci Neuropathol. 2006; 18: 73-76.

32. Doty RL. The olfactory vector hypothesis of neurodegenerative disease: it is viable? Ann Neurol. 2008; 63: 7-15.

\section{G. Brand}

Laboratoire de Neurosciences

Université de Franche-Comté

Place Leclerc 25000

Besançon

France

E-mail: gerard.brand@univ-fcomte.fr 